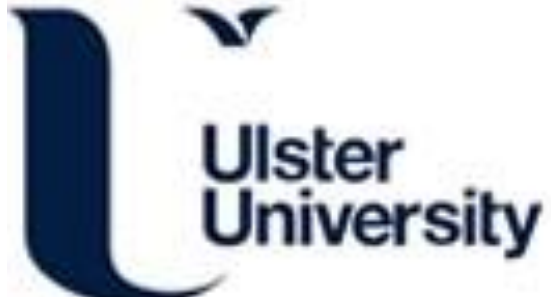

\section{Intracardiac impedance response during acute AF internal cardioversion using novel rectilinear and capacitor-discharge waveforms.}

Rababah, AS., Walsh, SJ., Manoharan, G., Walsh, PR., \& Escalona, OJ. (2016). Intracardiac impedance response during acute AF internal cardioversion using novel rectilinear and capacitor-discharge waveforms. Physiological Measurement, 37(7), 1129-1145. https://doi.org/10.1088/0967-3334/37/7/1129

Link to publication record in Ulster University Research Portal

\section{Published in:}

Physiological Measurement

Publication Status:

Published (in print/issue): 01/07/2016

DOI:

10.1088/0967-3334/37/7/1129

\section{Document Version}

Author Accepted version

\section{General rights}

Copyright for the publications made accessible via Ulster University's Research Portal is retained by the author(s) and / or other copyright owners and it is a condition of accessing these publications that users recognise and abide by the legal requirements associated with these rights.

\section{Take down policy}

The Research Portal is Ulster University's institutional repository that provides access to Ulster's research outputs. Every effort has been made to ensure that content in the Research Portal does not infringe any person's rights, or applicable UK laws. If you discover content in the Research Portal that you believe breaches copyright or violates any law, please contact pure-support@ulster.ac.uk. 


\title{
Intracardiac impedance response during acute AF internal cardioversion using novel rectilinear and capacitor- discharge waveforms
}

\author{
AS Rababah, ${ }^{1}$ SJ Walsh, ${ }^{2}$ G Manoharan, ${ }^{2}$ PR Walsh, ${ }^{1}$ OJ Escalona ${ }^{1, *}$ \\ ${ }^{1}$ School of Engineering, Engineering Research Institute, Ulster University, UK \\ ${ }^{2}$ Belfast Health and Social Care Trust, Royal Victoria Hospital, Belfast, UK \\ * Correspondence e-mail: oj.escalona@ulster.ac.uk
}

\begin{abstract}
Intracardiac impedance (ICI) is a major determinant of success during internal cardioversion of atrial fibrillation (AF). However, there have been few studies that have examined the dynamic behaviour of atrial impedance during internal cardioversion in relation to clinical outcome. In this study, voltage and current waveforms captured during internal cardioversion of acute AF in ovine models using novel radiofrequency $(\mathrm{RF})$ generated low-tilt rectilinear and conventional capacitordischarge based shock waveforms were retrospectively analysed using a digital signal processing algorithm to investigate the dynamic behaviour of atrial impedance during cardioversion. The algorithm was specifically designed to facilitate the simultaneous analysis of multiple impedance parameters, including: mean intracardiac impedance $\left(\mathrm{Z}_{\mathrm{M}}\right.$ ), intracardiac impedance variance (ICIV) and impedance amplitude spectrum area (IAMSA) for each cardioversion event. A significant reduction in ICI was observed when comparing two successive shocks of increasing energy where cardioversion outcome was successful. In addition, ICIV and IAMSA variables were found to inversely correlate to the magnitude of energy delivered; with a stronger correlation found to the former parameter. In conclusion, ICIV and IAMSA have been evidenced as two key dynamic intracardiac impedance variables that may prove useful in better understanding of the cardioversion process and that could potentially act as prognostic markers with respect to clinical outcome.
\end{abstract}

Keywords: atrial fibrillation, AF, acute AF model, internal cardioversion, intracardiac impedance variance, ICIV, impedance amplitude spectrum area, IAMSA, defibrillation.

\section{Introduction}

Atrial fibrillation (AF) is a growing public health problem affecting $0.65 \%$ of people aged 45-65 years old, and 9\% of those aged more than 80 years old (Roger et al. 2012). It is responsible for a major clinical and public health impact and substantial morbidity and mortality (Chamberlain et al. 2015). It causes a 3 to 5 fold increased risk of stroke (Wolf et al. 1991). In addition, AF is associated with a $20 \%$ increase in the length of hospital stay (Jorgensen et al. 1996). The need for improved management strategies and therapies therefore remains self-evident. 
It is widely accepted that the success of internal cardioversion of AF is determined by waveform related factors such as voltage, duration, tilt, as well as patient related factors including intracardiac impedance (ICI). Waveform related factors have been investigated intensively and multiple clinical studies to optimize the waveform for internal cardioversion of AF have been reported (Kodoth et al. 2011, Santos et al. 2003). However, little work has examined dynamic changes in the ICI as a determinant of shock success. Recently, Escalona and colleagues carried out a comparative study of low tilt biphasic waveform (LTBW) and low tilt monophasic waveform (LTMW) on 30 patients with persistent AF. A step up protocol (50-300V) was adopted for internal cardioversion of patients. Voltage and current data were recorded using oscilloscope and current probe during the procedure (Escalona et al. 2012). Based on this data set, several dynamic impedance studies were undertaken. It was reported that for successful monophasic and biphasic cardioversion there was a significant reduction in ICI during the first three shocks; while a significant reduction during the first three shocks was absent in patients who failed to cardiovert (Walsh et al. 2013) while subsequent categorization and grouping of the patient dataset into 4 groups (biphasic - successful cardioversion, biphasic - failed cardioversion, monophasic - successful cardioversion, monophasic - failed cardioversion) determined that the same characteristics mapped into the frequency domain (Walsh et al. 2015).

In this work, a retrospective study to further investigate the dynamic response of ICI during internal cardioversion of acute AF in ovine models, including the development of an algorithm that can facilitate the study of the dynamic behaviour of ICI with respect to waveform type, defibrillation energy and cardioversion outcome, is reported.

\section{Methods}

\subsection{Experimental setup}

A retrospective study on the experimental data gathered by Walsh et al. (2006) to investigate the efficacy of novel RF generated low-tilt rectilinear waveforms versus conventional capacitor-discharge (C) based waveforms (Ventritex, HVS-02, Sunnyvale, CA, USA) in terminating induced acute atrial fibrillation in ovine models is reported. Eleven sheep (mean weight $65 \pm 12 \mathrm{~kg}$ ) were used in this study and all were handled in accordance with UK and European guidelines (during the study a single model (sheep \#6) expired due to unrelated causes). Anaesthesia was achieved with a $20-30 \mathrm{mg} / \mathrm{kg}$ bolus of intravenous pentobarbitone (Sagatal $60 \mathrm{mg} / \mathrm{mL}$, Rhone Merieux Ltd, Harlow, UK) with ventilation using room air (Model 16/24, Ideal Respirator, CF Palmer Ltd, London, UK). Anaesthesia was maintained with a continuous infusion of pentobarbitone $(7-15 \mathrm{mg} / \mathrm{kg} / \mathrm{h}$ titrated to maintain a steady plane of anaesthesia). Under fluoroscopic guidance, defibrillation catheters were positioned in the high lateral right atrium (active fixation, Perimeter RA 7205, InControl Inc., Redmond, WA, USA) and the distal coronary sinus (Perimeter CS 7109, InControl Inc.) then connected to the RF or Ventritex HVS-02 defibrillator. A third 5 French bipolar atrial-J temporary pacing catheter (Cordis Europa N.V., Roden, Netherlands) was introduced into the right atrium via the right external jugular vein and connected to the atrial stimulator. AF was subsequently induced by rapid atrial stimulation at $100 \mathrm{~Hz}$ and an amplitude of 5-10V (Grass S44 Stimulator, Quincey, Mass, USA). Once atrial fibrillation had been confirmed for $>15$ seconds, cardioversion was attempted; with the outcome deemed successful when a sinus rhythm was returned within 5 beats post shock delivery (Walsh et al. 2006). 


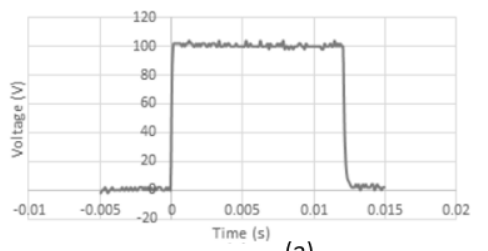

(a)

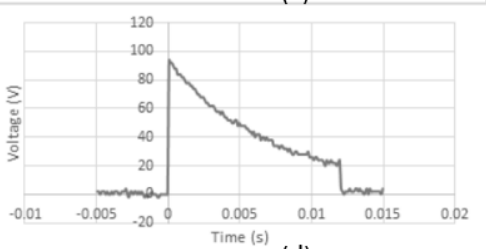

(d)

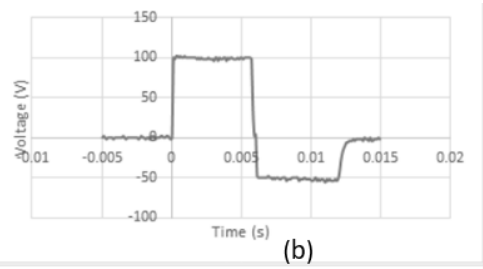

(b)

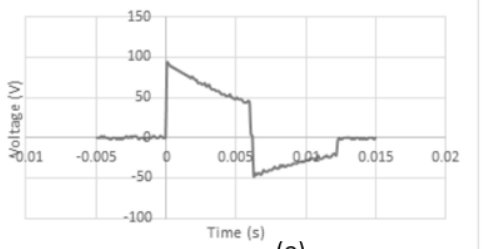

(e)

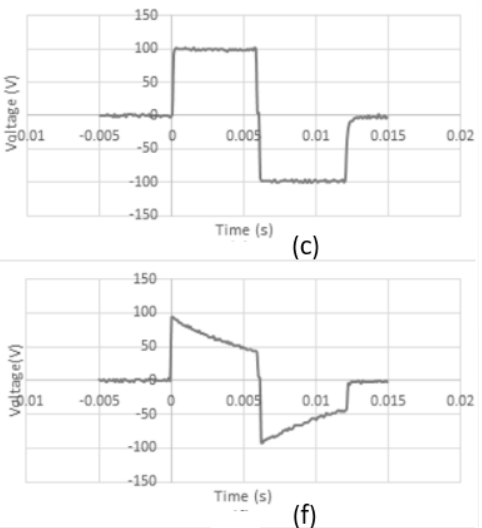

Figure 1: RF generated low-tilt rectilinear and conventional capacitor-discharge based cardioversion waveforms delivered to ovine models: (a) monophasic low-tilt rectilinear (12ms duration), (b) biphasic low-tilt rectilinear (12ms duration choronsymmetric amplitude asymmetric (phase 2 peak voltage = 50\% phase 1), (c) biphasic low-tilt rectilinear $(12 \mathrm{~ms}(6+6)$ duration amplitude symmetric (phase 2 peak voltage = $100 \%$ phase), (d) monophasic capacitor-discharge based conventional (12ms duration), (e) biphasic capacitor-discharge based conventional (12ms $(6+6)$ duration amplitude asymmetric (phase 2 peak voltage $=50 \%$ phase 1 ), (f) biphasic capacitor-discharge based conventional (12ms (6+6) duration amplitude symmetric (phase 2 peak voltage = 100\% phase 1)).

\subsubsection{AF cardioversion protocol}

With reference to figure-1, after induction of AF a single cardioversion shock waveform was chosen at random from one of seven cardioversion waveforms used in this study: RF generated low-tilt monophasic 100V (12ms duration), RF generated low-tilt biphasic 50/25V (12ms (6+6) duration amplitude asymmetric), RF generated low-tilt biphasic 100/50V (12ms (6+6) duration amplitude asymmetric), RF generated low-tilt biphasic 100/100V (12ms (6+6) duration amplitude symmetric), conventional monophasic $100 \mathrm{~V}$ (12ms duration), conventional biphasic 100/50V (12ms (6+6) duration amplitude asymmetric), conventional biphasic 100/100V (12ms (6+6) duration amplitude symmetric). Each waveform was consecutively delivered 5 times to each model; each model thereby receiving a total of 35 shocks. If cardioversion was successful, AF was re-induced within the model until the full protocol (35 shocks) was delivered. Voltage and current were measured directly from the output of the defibrillator at a sampling frequency of $10 \mathrm{kHz}$ using high voltage (V) and noncontact current probe (I) connected to a high-speed digital oscilloscope; with numerical values for both voltage and current with respect to time saved as a comma separated file (CSV file).

\subsection{Data processing and analysis algorithm}

With reference to figures 2 and 3 , recorded voltage and current data were exported from CSV format to MS-Excel format and loaded into MATLAB database for further analysis. A MATLAB algorithm was developed to automatically handle the large data files recorded; with case number was used to automate loading of the associated cardioversion waveforms and deduce the sampling frequency. In order to avoid spurious effects of electrical overshoot during the high speed rise / fall edges of the cardioversion waveform, data points captured within the rise / fall time were intentionally excluded from analysis. With reference to figure 4 , the algorithm was therefore designed to select and segment the waveform phase by windowing (positive $(1-5 \mathrm{~ms})$ or negative part $(7-11 \mathrm{~ms})$ of the signal for biphasic waveforms and / or early (1-5ms) or late part (7-11 ms) of the signal for monophasic waveforms. 


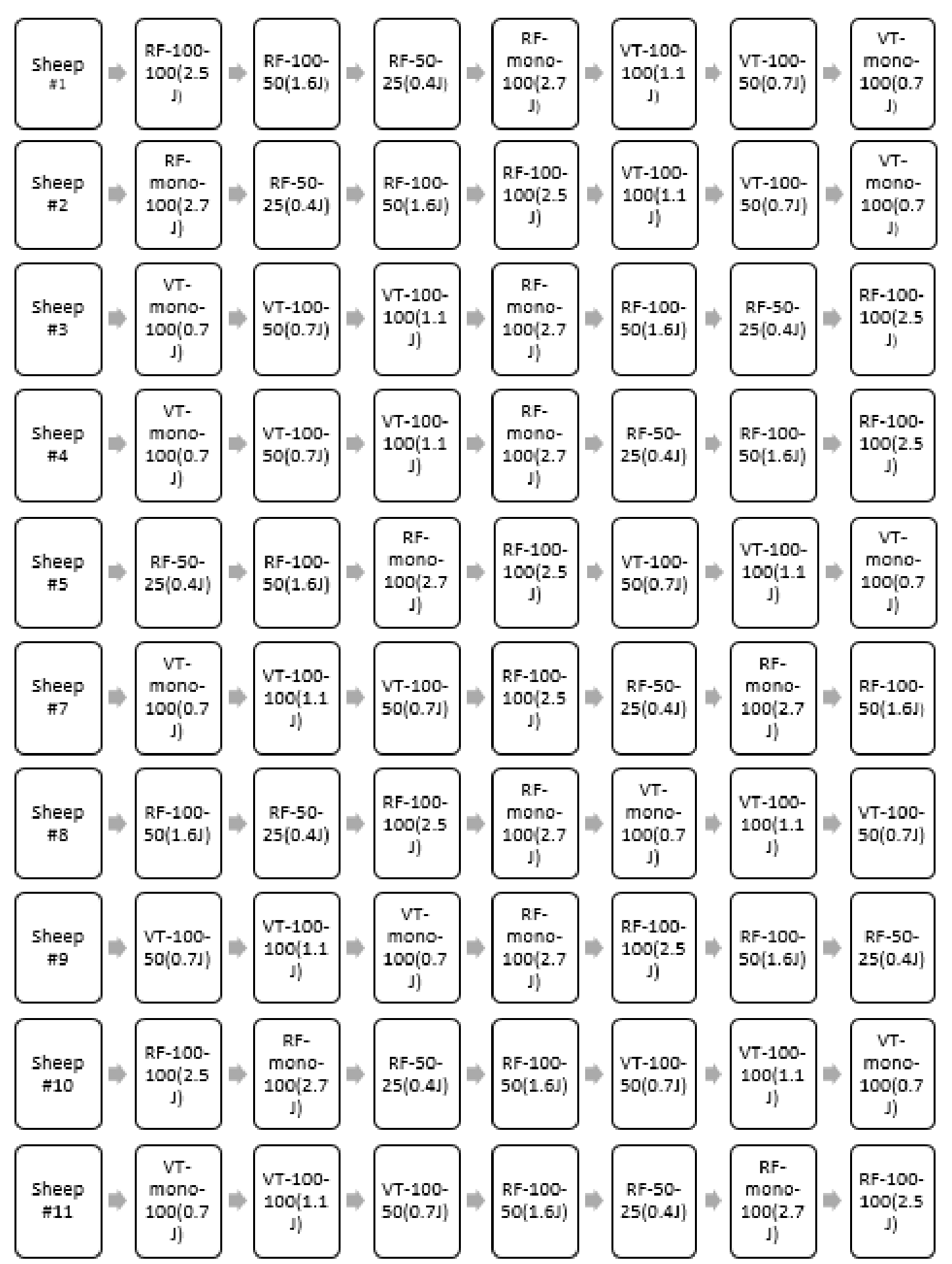

Figure 2: Sequencing and energy in Joules (J) of cardioversion waveforms delivered to each ovine model; where each waveform was consecutively delivered 5 times to each model with each model receiving a total of 35 shocks. 


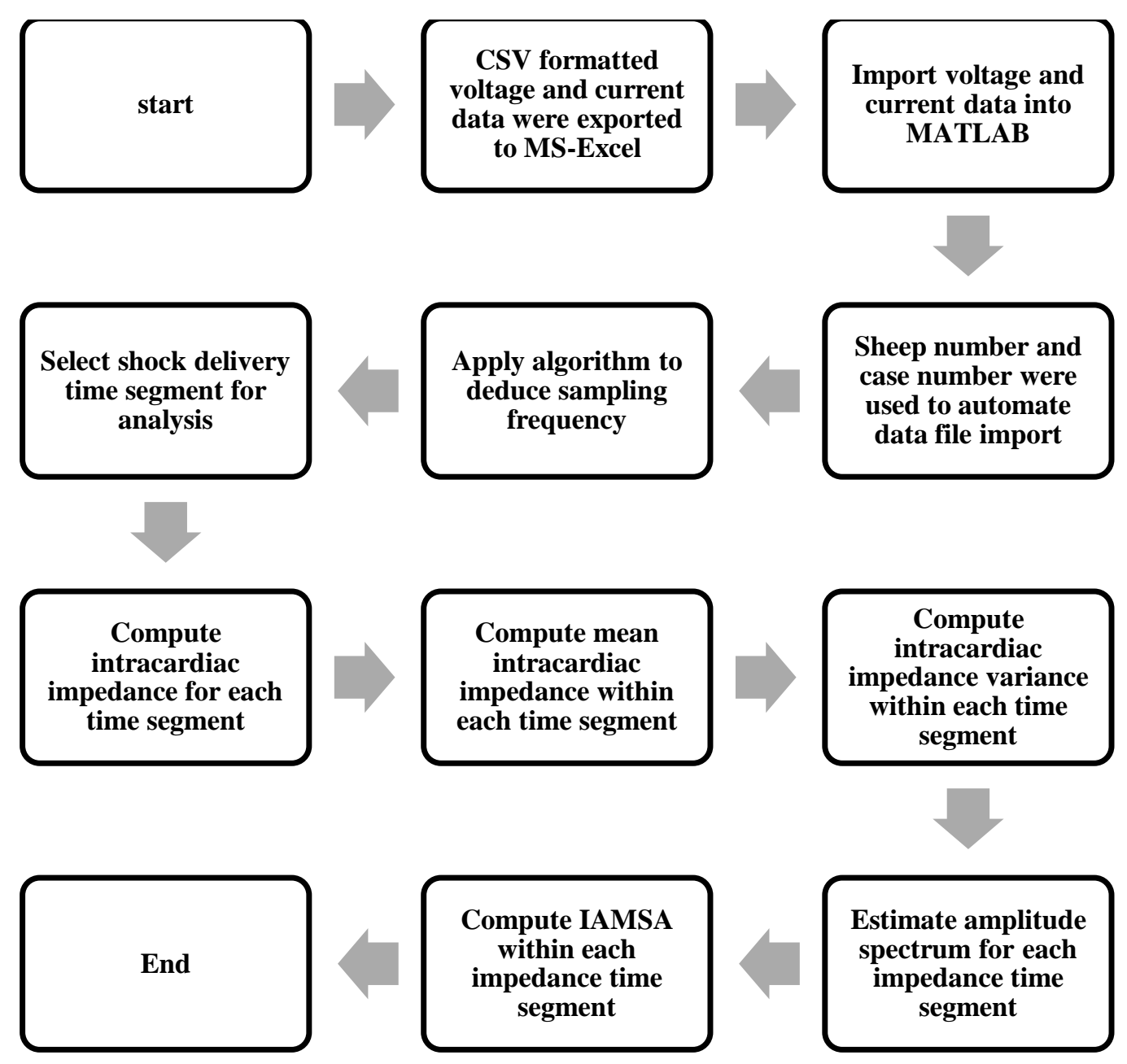

Figure 3: MATLAB flowchart for processing and analysis of defibrillation shock waveforms. 


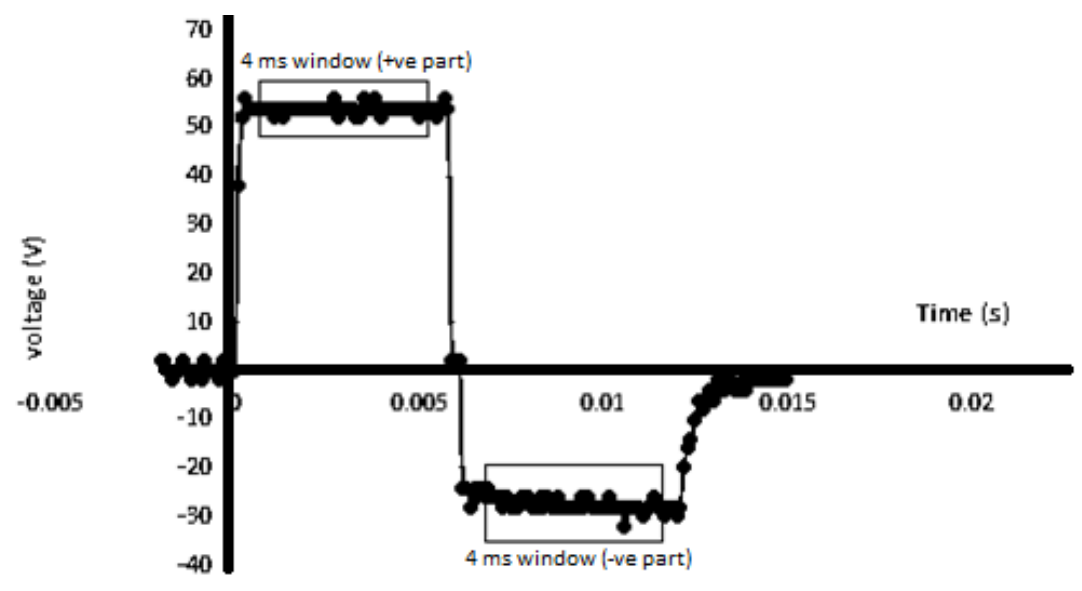

Figure 4: Voltage versus time plot of an RF generated low-tilt biphasic 50/25V waveform $(12 \mathrm{~ms}(6+6)$ duration amplitude asymmetric); showing the waveform segments windowed for analysis (positive or negative part of $4 m s$ (equivalent to early or late part in case of monophasic waveforms)).

\subsubsection{Intracardiac Impedance}

Based on the identified time segments of voltage and current data, the intracardiac impedance was calculated at each point as:

$$
\text { Impedance }(i)=\frac{\text { Voltage }(i)}{\text { Current }(i)}
$$

Where i denotes a range of $0-40$ sample points within the $4 \mathrm{~ms}$ window (see figure 4 ).

The algorithm was designed to subsequently compute three parameters, (i) mean intracardiac impedance $\left(\mathrm{Z}_{\mathrm{M}}\right)$, (ii) intracardiac impedance variance (ICIV) and (iii) impedance amplitude spectrum area (IAMSA)for positive and negative (early and late in case of monophasic waveforms) part of each cardioversion event as next described.

\subsubsection{Mean Intracardiac Impedance $\left(\mathrm{Z}_{\mathrm{M}}\right)$}

Mean intracardiac impedance, $Z_{M}$, within the defined positive and negative $4 \mathrm{~ms}$ windows was calculated as:

$$
Z_{\mathrm{M}}=\frac{\sum_{i=1}^{i=350} \text { Impedance }(i)}{350}
$$

This operation was repeated for each of 35 shocks delivered to each of 10 models; for a total of $\mathrm{n}=350$ calculated values of $\mathrm{Z}_{\mathrm{M}}$ within this study. 


\subsubsection{Intracardiac impedance variance (ICIV)}

Intracardiac impedance variance, ICIV, was mathematically calculated by averaging the squared differences for each $\mathrm{Z}$ data set as:

$$
\mathrm{ICIV}=\frac{\sum_{i=1}^{i=350}(\text { Impedance }(i)-Z \mathrm{M})^{2}}{350}
$$

Where, this parameter therefore describes the dispersion of impedance points within the specified time window.

\subsubsection{Impedance amplitude spectrum area (IAMSA)}

For IAMSA analysis, once the average impedance was calculated it was subtracted from each point in the impedance waveform to eliminate the dc component and obtain an impedance waveform that describes the change in impedance in a particular time segment (Walsh et al. 2015). The resulting waveform that represents the change in impedance in the time domain was then multiplied by a Hanning window of the same length to mitigate unwanted side lobe effects. The superiority of Hanning window over other windows in reducing the effect of side lobes while maintaining an appropriate frequency resolution when it was used as a part of algorithm to estimate the impedance spectrum was reported in our earlier work (Escalona et al 2015); where it is evidenced that since the intracardiac impedance spectrum contains very closely spaced features, a Hanning window with a narrower main lobe can perform better in estimating the spectrum. Next, a fast Fourier transform (FFT) algorithm was used to implement the frequency domain analysis; where, in order to obtain the correct amplitude values after applying the FFT function, the output amplitude spectrum was scaled by dividing the value of amplitude spectrum over half the number of points to return an amplitude spectrum which has the true amplitude values of the intracardiac impedance spectrum. Finally, IAMSA values were calculated as per equation 4 ; with $n=400$ data points yielding a spectral resolution of 25 $\mathrm{Hz}$ across a frequency range of $0-5000 \mathrm{~Hz}$.

$$
I A M S A=\sum_{i=1}^{i=200} A i * F i
$$

Where: $A$ denotes amplitude, $F$ denotes frequency, and i denotes a range of 1-200.

Figure 5 presents a block diagram of the MATLAB implementation of the IAMSA analysis algorithm. 


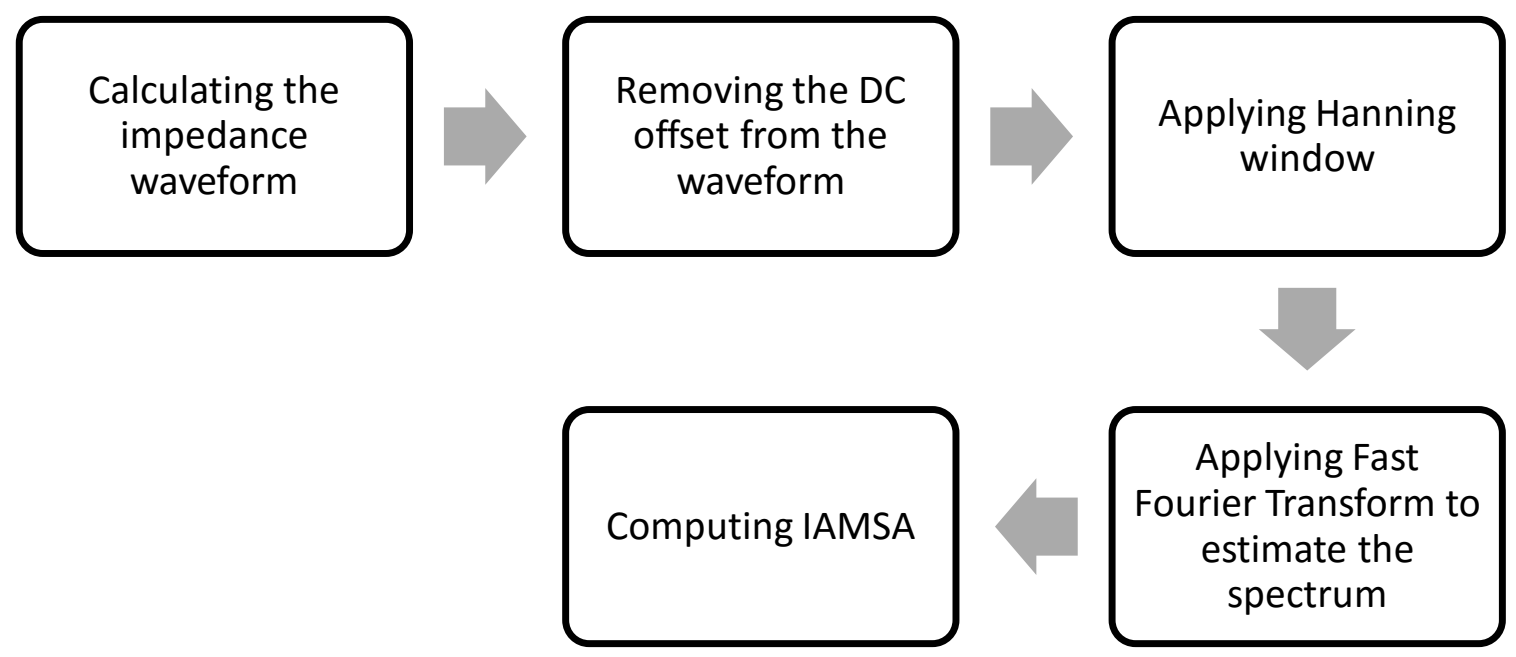

Figure 5: Block diagram of MATLAB implementation of IAMSA analysis algorithm.

The variations in impedance parameters: $\mathrm{Z}_{\mathrm{M}}$, ICIV, and IAMSA between two successive shocks of increasing or decreasing energy delivered were then compared to investigate the effect of change in energy delivered on each of these parameters. In addition, the effect of change in energy within each shock delivered on ICIV and IAMSA values was investigated in each shock.

\section{Results}

The percentage of success across all cardioversion waveforms in each sheep model is shown in table 1. A standard student t-test (two-tailed, paired distribution) was used to investigate the significance of variation in $\mathrm{Z}_{\mathrm{M}}$, ICIV, and IAMSA between two successive shocks of increasing or decreasing energy delivered with differences considered significant for $\mathrm{p}<0.01$. Table 2 tabulates the changes in $\mathrm{Z}_{\mathrm{M}}$, ICIV, and IAMSA between two successive shocks of increasing or decreasing energy delivered.

\subsection{Mean intracardiac impedance $\left(\mathrm{Z}_{\mathrm{M}}\right)$}

When comparing $Z_{M}$ of shock 5 of a specific waveform with $Z_{M}$ of shock 1 of the subsequent shock delivered, it is notable that there is a significant decrease in $Z_{M}$ between shocks when there is an increase in the delivered energy in a sheep case with a success rate of more than $80 \%$ (table $1 \& 2)\left(\Delta Z_{M}=-1.42 \Omega(\mathrm{SEM}=0.32), \mathrm{n}=21, \mathrm{p}=0.0004\right)$; where SEM is the standard error of mean, $\mathrm{n}$ is the number of shock pairs that are associated with an increase of energy. In addition, the change in $Z_{M}$ is inconsistence in sheep with low success rate such as sheep 5 and sheep $3\left(\Delta Z_{M}=-0.17 \Omega(S E M=0.38), n=6, p=0.7\right)$; where, again, $n$ is the number of shock pairs that are associated with an increase of energy. Moreover, inconsistency in the change of $Z_{M}$ occurs also when comparing two successive shocks associated with a decrease in the delivered energy $\left(\Delta \mathrm{Z}_{\mathrm{M}}=0.53 \Omega(\mathrm{SEM}=0.29), \mathrm{n}=29, \mathrm{p}=0.083\right)$ where $\mathrm{n}$ is the number of shock pairs that are associated with a decrease of energy (table 2 ). 
Table 1. Percentage success in each sheep model.

\begin{tabular}{|l|l|l|l|}
\hline Sheep Number & \% Success & Sheep Number & \% Success \\
\hline Sheep 1 & 83 & Sheep 7 & 94 \\
\hline Sheep 2 & 100 & Sheep 8 & 94 \\
\hline Sheep 3 & 63 & Sheep 9 & 89 \\
\hline Sheep 4 & 80 & Sheep 10 & 74 \\
\hline Sheep 5 & 33 & Sheep 11 & 80 \\
\hline
\end{tabular}

Table 2. Changes in $\mathrm{Z}_{\mathrm{M}}$, ICIV, and IAMSA between two successive shocks of increasing or decreasing energy delivered.

\begin{tabular}{ccc}
\hline $\begin{array}{l}\text { Intracardiac impedance } \\
\text { parameter }\end{array}$ & $\begin{array}{c}\text { Two successive shocks of } \\
\text { increasing energy }\end{array}$ & $\begin{array}{c}\text { Two successive shocks of } \\
\text { decreasing energy }\end{array}$ \\
\hline$\Delta \mathrm{Z}_{\mathrm{M}}$ & $-1.42 \pm 0.32$ & $0.53 \pm 0.29$ \\
$((\Omega \pm \mathrm{SEM}), \mathrm{p}$ value $)$ & $p=0.0004, \mathrm{n}=21$ & $p=0.083, \mathrm{n}=29$ \\
$\Delta \mathrm{ICIV}$ & $-6.57 \pm 0.71$ & $5.87 \pm 0.77$ \\
$\left(\left(\Omega^{2} \pm \mathrm{SEM}\right), \mathrm{p}\right.$ value $)$ & $p<0.0001, \mathrm{n}=27$ & $p<0.0001, \mathrm{n}=29$ \\
$\Delta \mathrm{IAMSA}$ & $-7772 \pm 1031$ & $7245.8 \pm 936.9$ \\
$((\Omega . \mathrm{Hz} \pm \mathrm{SEM}), \mathrm{p}$ value $)$ & $p<0.0001, \mathrm{n}=27$ & $p<0.0001, \mathrm{n}=29$ \\
\hline
\end{tabular}



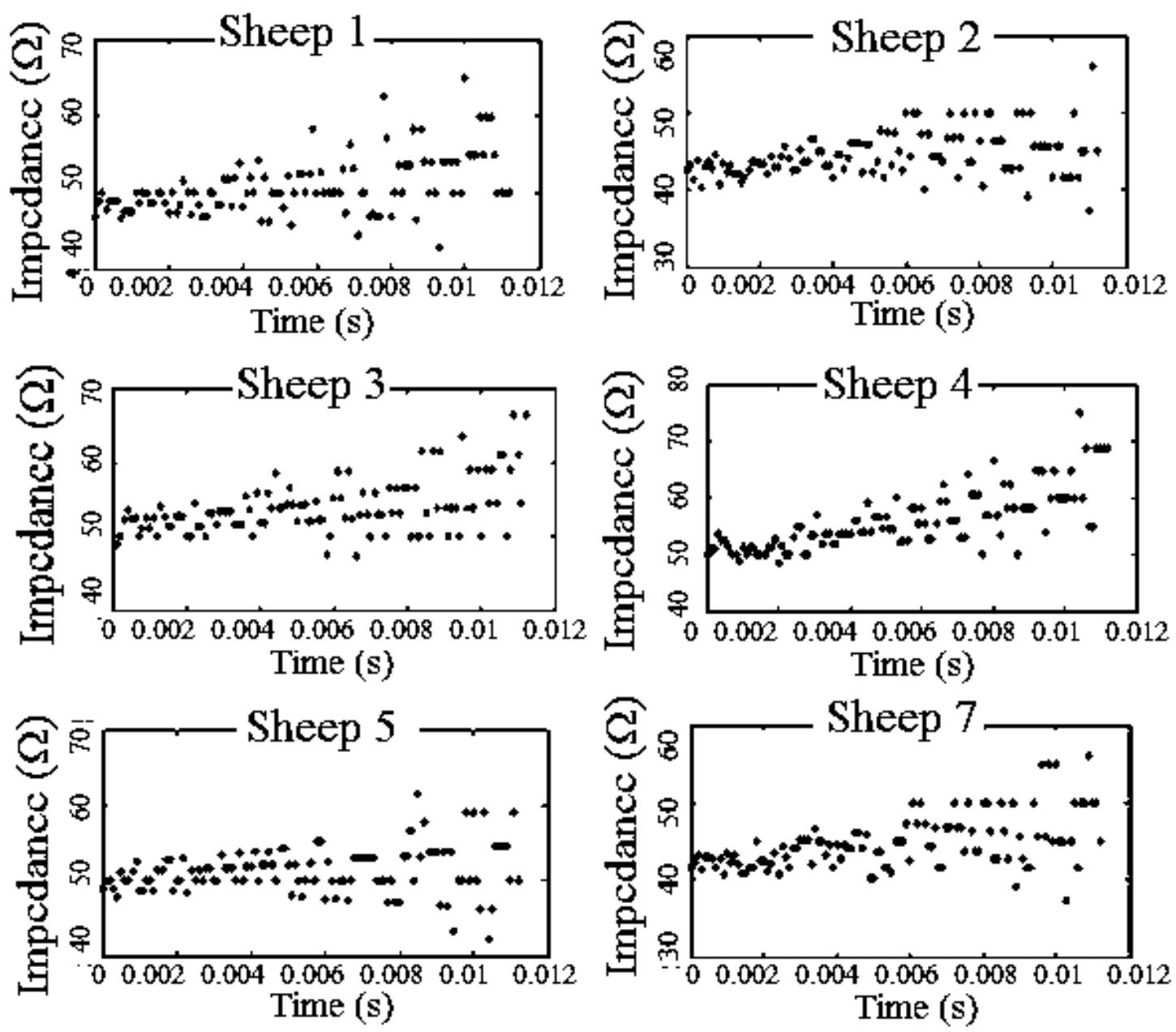

Figure6: Impedance data points during C-mono (conventional monophasic) shocks in 6 sheep models.

\subsection{Intracardiac impedance variance (ICIV)}

Similarly, ICIV was compared between two successive shocks that were associated with increase or decrease in the delivered energy. With reference to table 2, it is evident that wherever there is an increase in energy between any two successive shocks, there was a corresponding decrease in ICIV; where $\triangle \mathrm{ICIV}=-6.57, \mathrm{SEM}=0.71, \mathrm{n}=27, p<0.0001$; where SEM is the Standard Error of Mean, $\mathrm{n}$ is the number of shock pairs that are associated with an increase of energy (table 2). Conversely, a decrease in the energy was associated with an increase in ICIV $(\triangle \mathrm{ICIV}=5.87, \mathrm{SEM}=0.77, \mathrm{n}=29, p<0.0001$; again where $\mathrm{n}$ is the number of shock pairs that are associated with a decrease of energy (table 2 ). 
Sheep8: RF-50-25

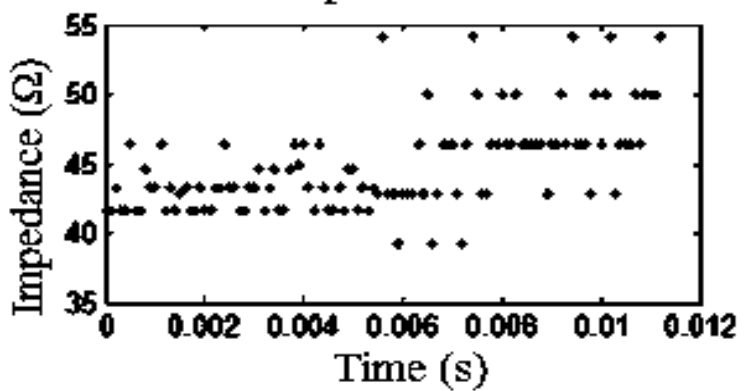

Sheep10: RF-100-100

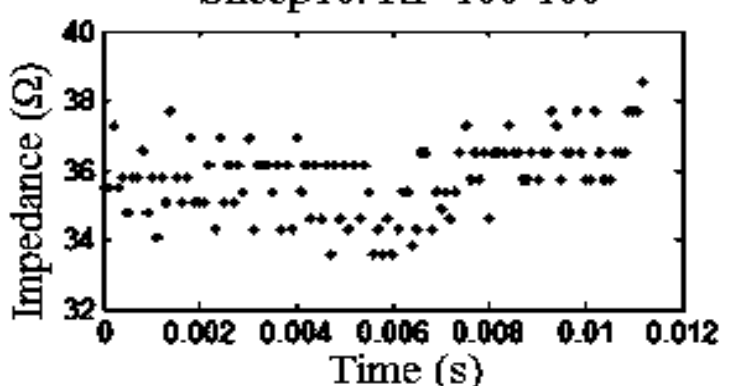

Sheep11: C-100-100

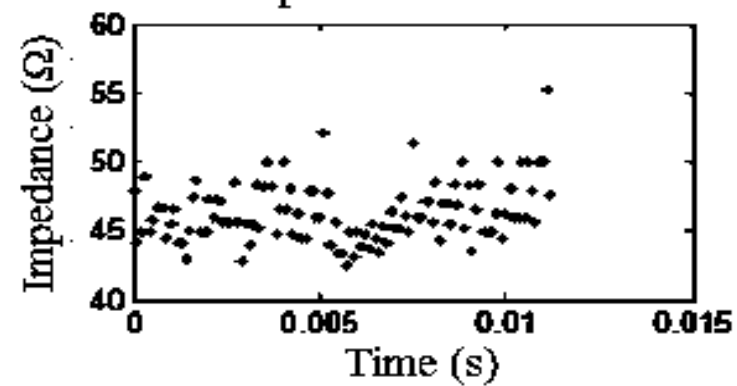

Sheep9: RF-100-50
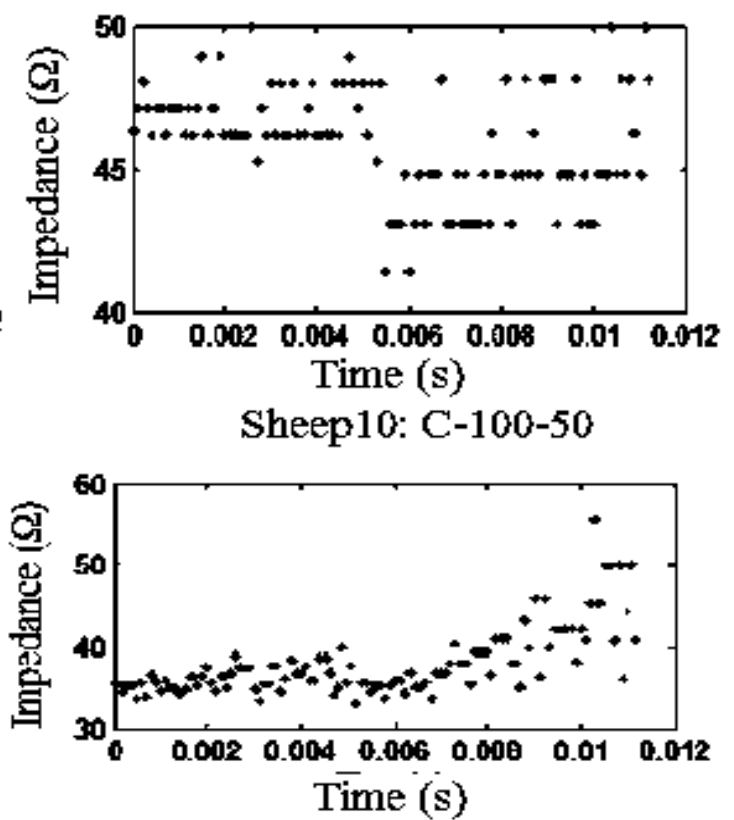

Sheep11: RF-Mono

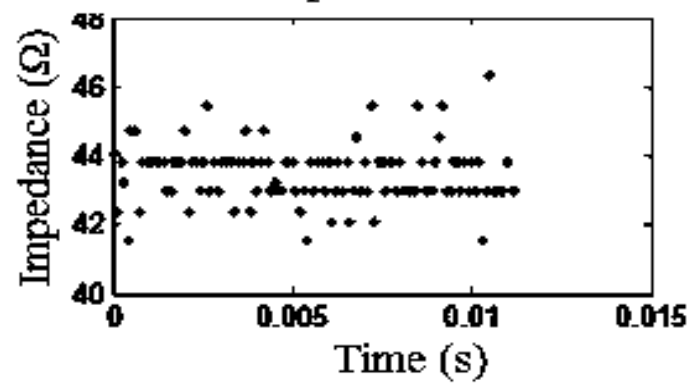

Figure7: Impedance data points during RF-50/25V, RF-100/50V, RF-100/100V, C-100/50V, C-100/100 and RF-Mono shocks in different sheep models $(R F=$ radiofrequency generated low-tile rectilinear cardioversion waveform, $C=$ conventional capacitor-based discharge cardioversion waveform)..

Furthermore, for conventional monophasic waveform shocks it was observed that during each of the cardioversion event - as voltage amplitude decreases (figure 1d), the energy delivered decreases while the impedance variance increases (figure 6). Comparable results were found when analysing the change in voltage amplitude (and consequently the change in energy) during other types of defibrillation shocks with respect to impedance variance; ICIV was higher for the positive phase of RF-50/25V, RF-100/50V and C-100/50V than for the negative phase (figure7). Also, this phenomenon of increasing ICIV with decreasing energy was consistently observed for all shocks of different waveforms types and in different ovine models.

\subsection{Impedance amplitude Spectrum Area (IAMSA)}

Impedance Amplitude Spectrum Area (IAMSA), which represents the amplitude spectrum over 
the range of frequency from $0-5000 \mathrm{~Hz}$, was calculated by summing the multiplications of frequency with the amplitude spectrum at this frequency; and which is an adapted parameter definition from the IAMSA presented by Walsh et al. (2015). Comparing the values of IAMSA between two successive shocks when there is an increase or decrease in energy during the whole protocol revealed that whenever there was a decrease in energy between two consecutive shocks, there was an increase in IAMSA value. In this study, there were 29 adjacent shock pairs associated with a decrease in energy during the entire protocol; presenting an average IAMSA decrease of: $\triangle \mathrm{IAMSA}=7245.8 \Omega . \mathrm{Hz}, \mathrm{SEM}=936.9, \mathrm{n}=29, p<0.0001$ (table 2). Conversely, an increase in energy in consecutive shocks resulted in a decrease in IAMSA value. The average increase of IAMSA presented by 27 adjacent shocks occurrences with an increase in energy was $\triangle \mathrm{IAMSA}=-7772 \Omega . \mathrm{Hz}, \mathrm{SEM}=1031, \mathrm{n}=27, p<0.0001$ (table 2).

Finally, figure 8 shows the impedance across frequency response for 4 different types of RF generated low-tilt rectilinear waveforms; RF-50/25V (figure $8 \mathrm{a}$ and $8 \mathrm{~b}$ ), RF-100/50V (figure 8c and $8 \mathrm{~d}$ ), RF-100/100V (figure 8e and $8 \mathrm{f}$ ) and RF-mono (figure $8 \mathrm{~g}$ and $8 \mathrm{~h}$ ). In figures $8 \mathrm{a} / \mathrm{b}$, $8 \mathrm{c} / \mathrm{d}, 8 \mathrm{e} / \mathrm{f}$ and $8 \mathrm{~g} / \mathrm{h}$, the voltage amplitudes are 25, 50, 100 (all biphasic) and $100 \mathrm{~V}$ (monophasic) with corresponding delivered energies of $0.08,0.31,1.25,1.25 \mathrm{~J}$, respectively. Upon inspection, it is evident that as the voltage amplitude increases the delivered energy also increases (area under the curve decreases). It is also of interest to note that the third and fourth rows in figure 8 (e/f and $\mathrm{g} / \mathrm{h}$ ) show approximately equal areas under each curve; as the voltage amplitude and delivered energy are equivalent for each of these cardioversion shock waveforms. The same behaviour was found when comparing the impedance frequency response of the 3 types of conventional waveforms, C-100V (monophasic), C-100/50V (biphasic) and C-100/100 (biphasic); where all have approximately the same voltage amplitude and delivered energy in the first phase of the waveform and consequently the area under curves in figure $9 \mathrm{a}, 9 \mathrm{c}$, and $9 \mathrm{e}$ are approximately the same. However, these conventional waveforms arranged in ascending order in terms of voltage amplitude and delivered energy in the negative part (late part in case of monophasic) is C-mono-100, C-100/50V, C-100/100V (figure $1 \mathrm{~d}, 1 \mathrm{e}$, and 1f), and in terms of area under curve is C-100/100V, C-100/50V, C-mono- $100 \mathrm{~V}$ (figure $9 \mathrm{~b}$, 9d, 9f). Hence, the negative correlation between the delivered energy and IAMSA is again evidenced.

\section{Discussion}

As previously stated, it is widely accepted that the success of internal cardioversion of atrial fibrillation is influenced by waveform related factors such as voltage, duration and tilt, as well as patient related factors including intracardiac impedance (ICI). It has also been recognized that the ICI is one of the major determinants of the minimum energy required for successful internal cardioversion (Escalona et al 2012, Walsh et al. 2013, Walsh et al 2015). Hence, intracardiac impedance is an important factor that should be always considered in optimising the treatment protocol of internal cardioversion. The ICI changes continuously due to the movement of ions through ion channels imbedded in cell membrane in response to the applied voltage and current. It also changes as a result of both electrochemical and physiological mechanisms (KenKnight et al. 1995). In this study, several intracardiac impedance parameters including $\mathrm{Z}_{\mathrm{M}}$, ICIV, and IAMSA were investigated. Additionally, relationships between these parameters and other factors such as waveform type, defibrillation energy were also examined. Overall, the study revealed interesting results and findings that are in keeping with past studies and extend upon previous findings in respect of the novel RF generated low-tilt rectilinear 
waveforms used to cardiovert acute $\mathrm{AF}$ in ovine models. In addition, a new intracardiac impedance parameter (intracardiac impedance variance ICIV) was evidenced to inversely correlate to the magnitude of energy delivered and may also potentially serve as a prognostic marker to clinical outcome.

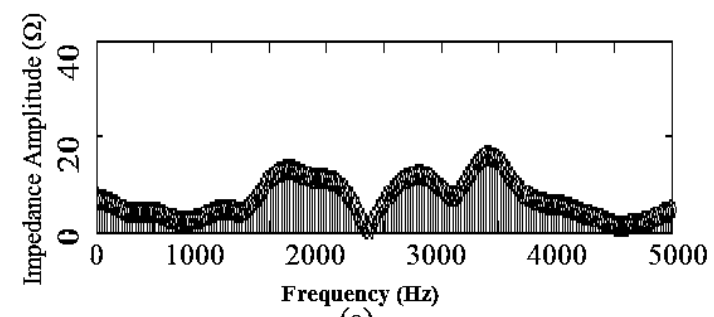

(a)

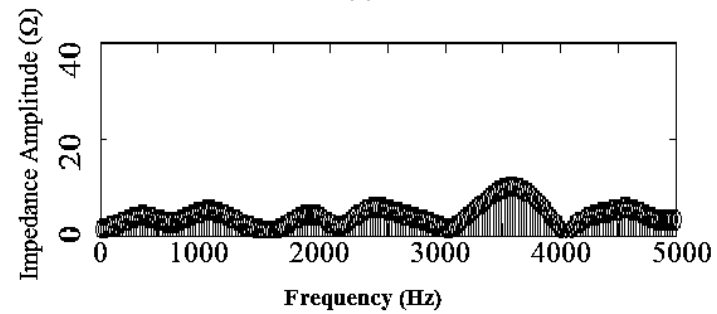

(c)

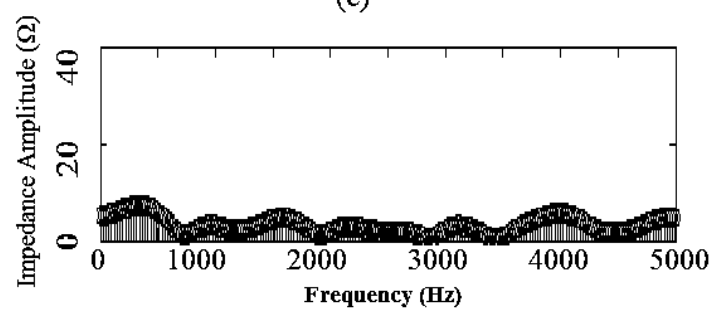

(e)

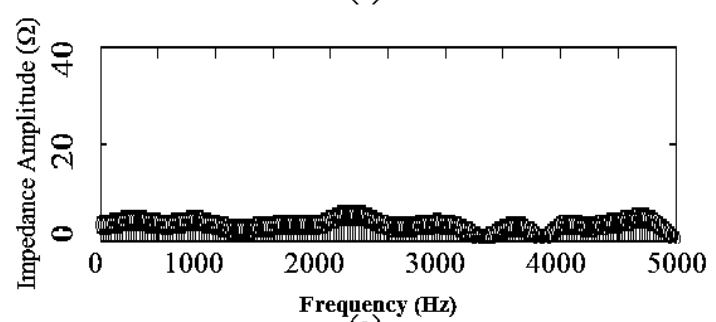

(g)

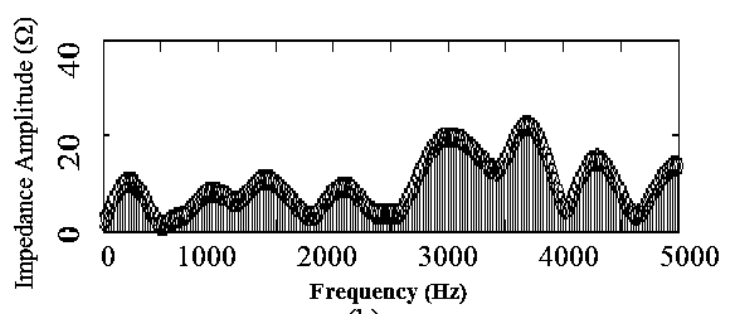

(b)

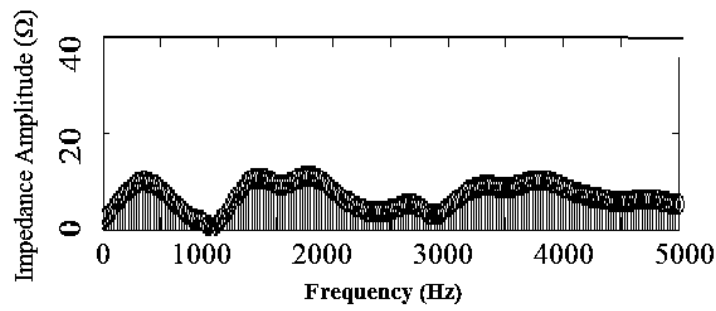

(d)

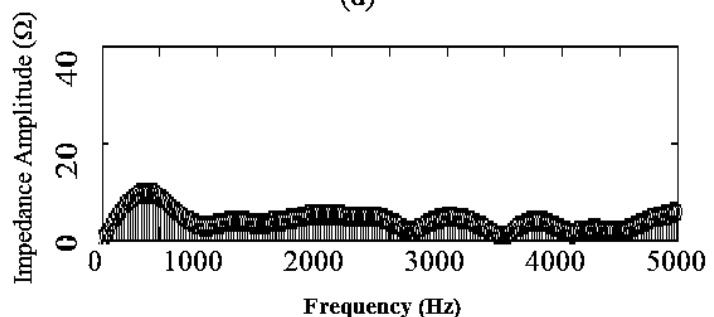

(f)

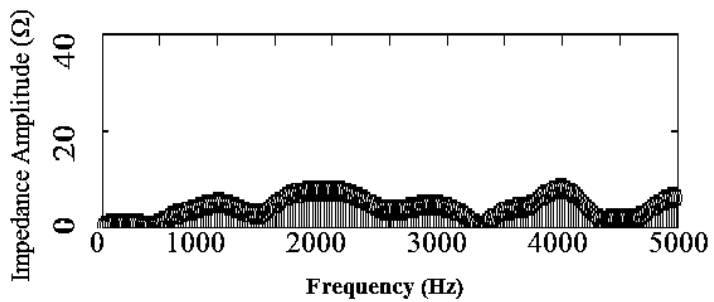

(h)

Figure 8: Impedance amplitude response across frequency during positive (early) duration ( $a, c, e, a$ and g) or negative (late) duration ( $b, d, f$, and $h)$ ) of RF-50/25V ( $a$ and b), RF-100/50V (c and d), RF-100/100V (e and f) and RF-mono (g and h) cardioversion waveforms. 


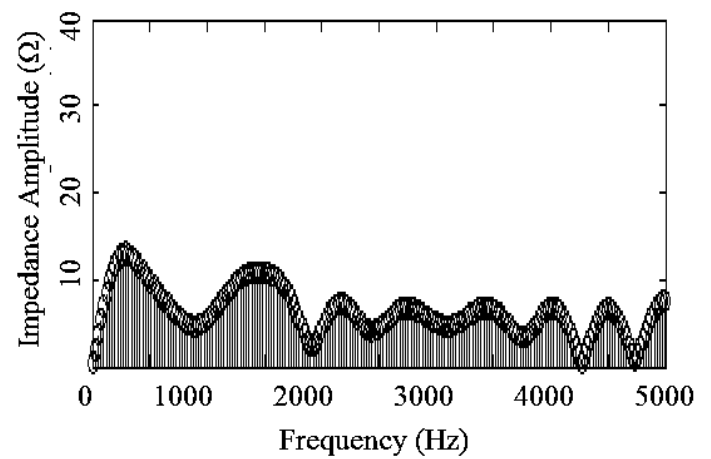

(a)

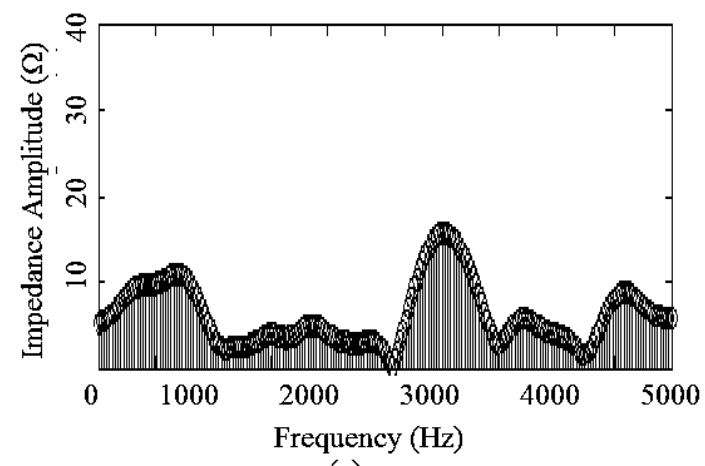

(c)

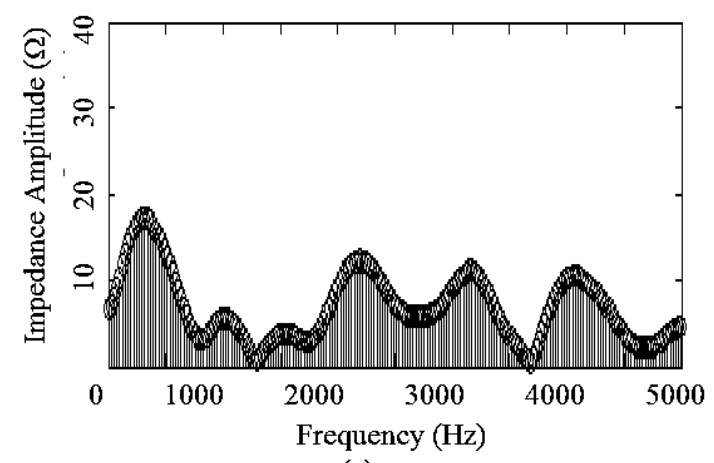

(e)

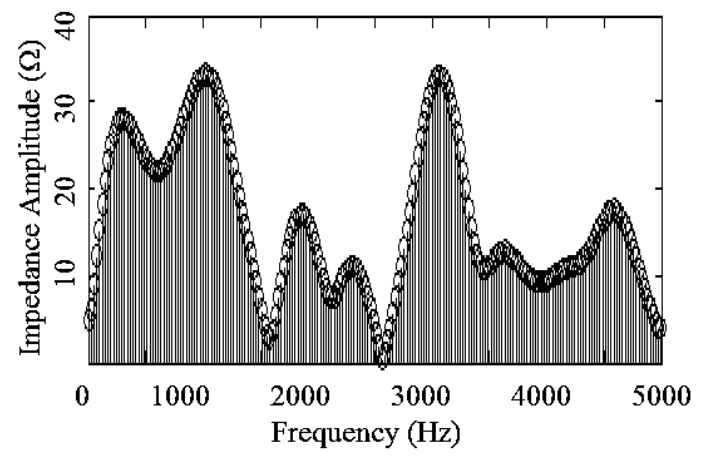

(b)

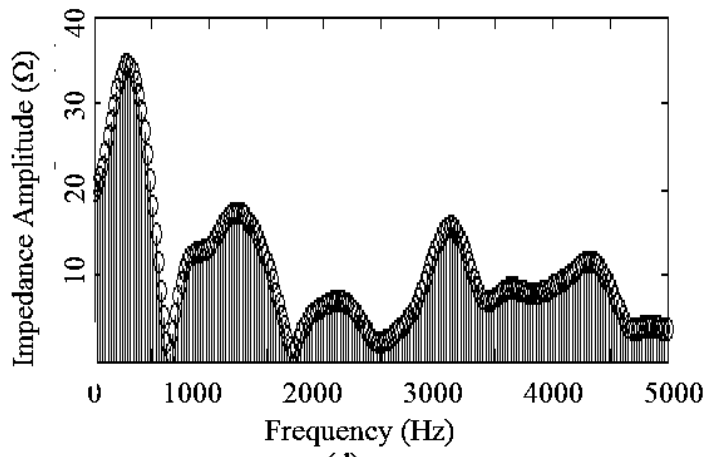

(d)

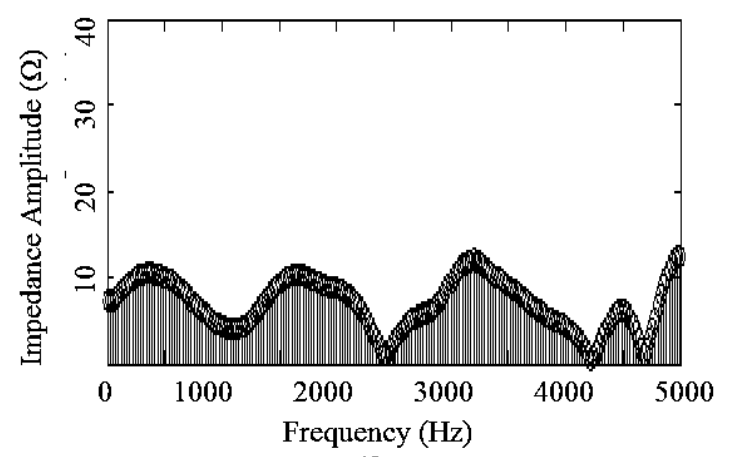

(f)

Figure 9: Impedance amplitude response across frequency during positive (early) duration (a, $c$ and e) or negative (late) duration ( $b$, $d$ and $f$ ) of C-mono-100V ( $a$ and b), C-100/50V ( $c$ and $d)$, and C-100/100V (e and f) waveforms.

\subsection{Mean intracardiac impedance $\left(\mathrm{Z}_{\mathrm{M}}\right)$}

In respect of the mean intracardiac impedance $\left(\mathrm{Z}_{\mathrm{M}}\right)$, is was observed that whenever there was an increase in energy between two successive shocks it was accompanied by a significant decrease in this parameter. Interestingly, a significant decrease in the $\mathrm{Z}_{\mathrm{M}}$ was absent in sheep 3 and 5; which also had a very low percentage of success. Furthermore, there are significant similarities in results with those reported by Walsh et al. (Walsh et al. 2015); who studied the dynamic changes in ICI during internal cardioversion of atrial fibrillation in humans when applying a step-up energy cardioversion shock protocol (Walsh et al. 2013). This work reported 
that there was a significant linear decrease ICI for the first three shocks in patients who successfully cardioverted irrespective of cardioversion waveform used (monophasic or biphasic low-tilt rectilinear). However, for patients who failed to cardiovert, the decrease in impedance was insignificant. Nevertheless, there are two key differences between the two studies. Firstly, in this present study, acute AF models were studied using a randomised protocol (i.e. the seven waveforms that delivered different energies were applied to each sheep in random order). This effectively means that while sometimes there was an increase in energy at other times there was a decrease in energy between two successive shocks. In contrast, in the study performed by Walsh et al. (2013), a consistent step up energy cardioversion shock protocol was adopted; where the voltage amplitude was increased from $50-300 \mathrm{~V}$ in a $50 \mathrm{~V}$ steps and accordingly the cardioversion energy delivered was always increased. Secondly, in this present study, different waveform types (monophasic and biphasic RF generated low-tilt rectilinear and conventional capacitor discharge based cardioversion waveforms) were applied during the procedure while only monophasic and biphasic RF generated low-tilt rectilinear waveforms were used in Walsh's study (Walsh et al. 2013). As a consequence of keeping all other variables constant and changing one parameter, which is the delivered energy, in Walsh's study, the reduction in impedance between the successive shocks was more significant than in the present study. However, that said and in spite of inherent aforementioned limitations, in this study the reduction in ICI was significant between any two successive shocks that were associated with an increase in energy. This finding explains the possible advantage of energy step up protocol in AF cardioversion in reducing the energy threshold over the alternative strategy of starting with high energy shock; where the first shock is suspected of having a favourable effect on the cardiac substrate for the next shock by reducing intracardiac impedance consequently enabling an increased current density to be delivered during the next cardioversion attempt. A reduction in transthoracic impedance as a result of repeated counter shocks has long been described in the literature. Dahl et al. (1976) reported a progressive decrease in the transthoracic impedance as a consequence of repeated counter shocks. They also studied the effect of time interval between counter shocks and found that the 3 minute interval was associated with the greatest reduction in transthoracic impedance among all other intervals. This behaviour of reduced transthoracic impedance after each shock somehow prepares the cardiac tissue to be affected more by the next shock and consequently leads to a higher percentage of success of the next shock. Recent studies also suggest that low energy multi-stage defibrillation therapy to terminate atrial fibrillation with less energy than a single shock. Janardhan et al. (2014) showed on canine model that low energy multi-stage defibrillation therapy significantly lowered AF defibrillation threshold (Janardhan et al. 2014). Another study by Li et al. (2011) revealed that three stage electrotherapy is the optimum in term of reducing defibrillation threshold. All these reports would be in support of the antifibrillatory pacing electrotherapy of AF ( $\mathrm{Li}$ et al. 2011), which could be implemented by new implantable smart pacemakers.

\subsection{Intracardiac impedance variance (ICIV)}

In respect of the intracardiac impedance variance (ICIV), the most important finding to emerge was the strong correlation between ICIV and delivered energy. In almost every case where energy decreased between two successive shocks ICIV increased (and vice versa). The relationship between ICIV and energy delivered was very consistent in all shocks $(n=350)$ and in different ovine models for different cardioversion waveforms. This relationship is clearly revealed when studying this parameter in C-mono waveform where the energy decreased gradually with time (see figure 1d). This gradual fall in energy within a shock led to a gradual rise in ICIV accordingly (figure 6). Another example that highlights this association is the 
difference in ICIV between the positive and negative phase of RF-50/25V and RF-100/50V (figure 7). A two-fold decrease in voltage between the positive and negative phase led to a four-fold decrease in energy which in turn caused more than four-fold increase in ICIV in most of the shocks examined. To the best of our knowledge, the ICIV vs energy relationship has not been previously reported in the literature. Hence, the results from this study are a contribution to the literature in that ICIV within a shock is well correlated to the delivered energy level. A possible explanation for this phenomenon could be that when the cardiovesrion energy is increased, the current density delivered through the myocardium also increases. As it is the current (and current density) that cardioverts, an increase in the current density that flows through the myocardium leads to a higher number of synchronized cardioverted myocytes which cause a synchronized and organized transfer of ions and this organized ion current may give rise to a smaller variation of impedance within a shock. In contrast, lower energy leads to lower current (and current density) through the myocardium and consequently some of the cardiac cell cardiovert while the other remain in chaos. This would give rise to a change in ion transfer and consequently a higher change in impedance within a shock (ICIV).Considering this finding which shows that there is a very strong negative relationship between ICIV and delivered energy, and the fact that the effectiveness of internal cardioversion increases as the delivered energy increases, assuming that all other variables remain fixed it could be postulated that the success of internal cardioversion during the energy step up protocol is inversely related to ICIV. Nevertheless, this relationship between ICIV and energy (and consequently between ICIV and percentage success) demands a further study to investigate the change in ICIV between different shocks in patients who successfully cardioverted and who failed to cardiovert to understand more accurately the prognostic role of ICIV with respect to cardioversion outcome.

\subsection{Impedance Amplitude Spectrum Area (IAMSA)}

In respect of the intracardiac impedance amplitude spectrum area (IAMSA), measurement results revealed that while performing the procedure, whenever there was an increase in the energy between two successive shocks, there was a decrease in IAMSA and vice versa. Also, IAMSA values of RF-50/25V, RF-100/50V, C-100/50V, and C-mono-100V waveforms were higher in the second part of waveform (lower energy) than in the first part (higher energy) (figures 8 and 9). This means that IAMSA value is highly dependent on the delivered energy. In addition, IAMSA values were very high for C-mono-100v and C-100/5v0 because the very low value of energy delivered at the end of shock duration period. Walsh et al (2015) have studied IAMSA and reported that there is a significant reduction in IAMSA between the first (lower energy) and third (higher energy) shocks in all patients who cardioverted (equivalent to a decrease in IAMSA when there is an increase in energy) and this trend was also observed within this study. However, this study shows that this finding holds true for both rectilinear and capacitor based waveforms. The results on the IAMSA values have a similar trend to that of ICIV. The association of these parameters with energy was also similar. This could potentially be because the amplitude spectrum describes the variance of impedance over the frequency domain. However, the delivered energy was more associated with ICIV parameter than IAMSA. This could potentially mean that the ICIV parameter could potentially prove more useful in the development of new low energy antifibrillatory electrotherapies. In the literature, several studies showed the capability of ECG derived amplitude spectrum area (AMSA) to predict the success of defibrillation (Young et al. 2004; Ristagno et al. 2013; Wu et al. 2010; Indik et al. 2013, Howe et al. 2014). In these previous studies, AMSA values were calculated for VF waveforms and prior to the delivered shock, but in this study, it is calculated for the 
impedance waveform during the delivered shock. In the same line of providing decision support to maximise AF electrical cardioversion treatment success, IAMSA and ICIV parameters may be combined with ECG based frequency domain techniques (Alcaraz et al. 2009) and non-linear metrics (Alcaraz et al.2010), in order to potentially develop new low energy patient specific therapies with enhanced outcomes.

\section{Conclusions and future studies}

In this study, voltage and current waveforms captured during acute AF internal cardioversion using novel rectilinear and conventional capacitive-discharge electrical shock waveforms were used to develop digital signal processing algorithms that facilitated the investigation of the dynamic behaviour of atrial impedance during internal cardioversion of acute AF in the ovine model. The algorithm was specifically designed to analyse multiple parameters including: mean intracardiac impedance $\left(\mathrm{Z}_{\mathrm{M}}\right)$, intracardiac impedance variance (ICIV) and impedance amplitude spectrum area (IAMSA) for each cardioversion shock delivered. A statistically significant reduction in ICI was observed when comparing two successive shocks of increasing energy when the cardioversion outcome was successful. In addition, ICIV and IAMSA parameters were found to inversely correlate to the magnitude of energy delivered; with a stronger correlation found with the former parameter. In conclusion, ICIV and IAMSA have been evidenced as two key dynamic intracardiac impedance metrics that may prove useful in better understanding the cardioversion process and may potentially act as prognostic markers to clinical outcome; thereby helping to potentially facilitate new strategies in lowenergy (painless) AF antifibrillation cardiovserion and pacing therapies.

\subsection{Recommendations for further studies}

Future studies should consider investigating ICI, ICIV and IAMSA parameters in a study that follows an energy step up protocol using a single cardioversion waveform (to eliminate the effect of randomly changing waveform types) and thereby examine the impact of changing energy content more closely on each of the aforementioned parameters and with respect to cardioversion outcome. In the latter experimental setting, usefulness of time-variant and timeinvariant impedance attributes by means of vector impedance analysis techniques (Sanchez et al. 2015) could be further investigated.

\section{Acknowledgements}

During the original clinical model data gathering work, Dr Simon Walsh was the recipient of a Research and Development Fellowship from the Northern Ireland Health and Personal Social Services Office. Professor Omar Escalona dedication in this retrospective study was supported by philanthropic funds equally from the Ulster Garden Villages Ltd. and the McGrath Trust (UK).

\section{References}

Alcaraz R, Rieta JJ 2009. Time and frequency recurrence analysis of persistent atrial fibrillation after electrical cardioversion. Physiological Measurement 30(5): 479-89.

Alcaraz R, Rieta JJ 2010. The application of nonlinear metrics to assess organization differences in short recordings of paroxysmal and persistent atrial fibrillation. Physiological Measurement 31(1): 115-130. 
Chamberlain A M, Gersh B J, Alonso A, Chen L Y, Berardi C, Manemann S M, Killian J M, Weston S A and Roger V L 2015. Decade-long trends in atrial fibrillation incidence and survival: a community study. The Am. J. Med. 128: 260-7.

Dalzell G W, Anderson J and Adgey A A 1991. Factors determining success and energy requirements for cardioversion of atrial fibrillation: revised version. The Q. J. Med. 78: 85-95.

Dahl C F, Ewy G A, Ewy M D and Thomas E D 1976. Transthoracic impedance to direct current discharge: effect of repeated countershocks. Med. Instrum. 10: 151-4.

Escalona O, Kodoth V, Castro N, Xavier S, Walsh P, Glover B, Lau E and Manoharan G 2012. Linear variation analysis of intracardiac atrial impedance during internal cardioversion using rectilinear waveforms and energy step up protocol. Computing in Cardiology 39: 721-4.

Escalona O J, Walsh P R, Rababah A S, Kodoth V and Manoharan G 2015. Denoising RF defibrillator waveforms for intracardiac atrial substrate impedance characterization using digital filtering techniques. World Congress on Medical Physics \& Biomedical Engineering, IFMBE Proc. 51: 1329-32.

Howe A, Escalona OJ, Di Maio R, Massot B, Cromie NA, Darragh KM, Adgey J, McEneaney DJ 2014. A support vector machine for predicting defibrillation outcomes from waveform metrics. Resuscitation 85 (3): 343 - 349.

Indik J, Conover Z, McGovern M, Silver A, Spaite D W, Bobrow B J and Kern K B 2013. Amplitudespectral area is associated with survival from out-of-hospital ventricular fibrillation cardiac arrest. Circulation 128: A10855.

Janardhan A H, Gutbrod S R, Li W, Lang D, Schuessler R B and Efimov I R 2014. Multistage electrotherapy delivered through chronically-implanted leads terminates atrial fibrillation with lower energy than a single biphasic shock. J. Am. Coll. Cardiol..63: 40-8.

Jorgensen H S, Nakayama H, Reith J, Raaschou H O and Olsen T S 1996. Acute stroke with atrial fibrillation: the copenhagen stroke study. Stroke.27: 1765-69.

KenKnight B H, Eyüboğlu B M and Ideker R E 1995. Impedance to defibrillation countershock: does an optimal impedance exist? Pacing Clin. Electrophysiolog. 18: 2068-87.

Kodoth V, Castro N C, Glover B M, Anderson J M, Escalona O J, Lau E and Manoharan G 2011. Waveform optimization for internal cardioversion of atrial fibrillation. J. Electrocardiol..44: 689-93.

Li W, Janardhan A H, Fedorov V V, Sha Q, Schuessler R B and Efimov I R 2011. Low-energy multistage atrial defibrillation therapy terminates atrial fibrillation with less energy than a single shock. Circ. Arrhythm. electrophysiol. 4: 917-25.

Ristagno G, Li Y, Fumaqalli F, Finzi A and Quan W 2013. Amplitude spectrum area to guide resuscitation-a retrospective analysis during out-of-hospital cardiopulmonary resuscitation in 609 patients with ventricular fibrillation cardiac arrest. Resuscitation 84: 1697-703.

Roger V L et al. 2012. Heart disease and stroke statistics - 2012 update: a report from the American Heart Association. Circulation 125: e2-e220.

Sanchez B, Louarroudi E, Pintelon R 2015. Time-invariant measurement of time-varying bioimpedance using vector impedance analysis. Physiological Measurement 36 (3): 595-620. 
Santos J A, Walsh S, Evans E N, Escalona O, Manoharan G, Anderson J,Allen J D and Adgey A A 2003. Transdermal atrial defibrillation with bipolar low-tilt pulses. Electronic Letters 39: 1789-90.

Walsh P R, Escalona O J, Kodoth V, Castro N C, McEneaney D, Lau E and Manoharan G 2013. Dynamic changes in intracardiac resistance as a predictive marker during internal cardioversion of atrial fibrillation. Computing in Cardiology 40: 611-614.

Walsh P, Kodoth V, McEneaney D, Rodriques P, Velasquez J, Waterman N and Escalona O 2015. Towards low energy atrial defibrillation. Sensors 15: 22378-400.

Walsh S J, Manoharan G, Escalona O J, Evan N, Allen J D, Anderson J M and Adqey A A 2003. Transvenous cardioversion - a novel asymmetric rectangular biphasic waveform from a radiofrequency defibrillator compared with conventional waveforms in atrial fibrillation. J. Electrocardiol. 36: 191-2.

Walsh S J, Manoharan G, Escalona O J, Santos J, Evans N, Anderson J M, Stevenson M, Allen J D and Adgey A A 2006. Novel rectangular biphasic and monophasic waveformsdelivered by a radiofrequencypowered defibrillatorcompared with conventional capacitor-based waveforms in transvenous cardioversion of atrial fibrillation. Europace 8: 873-880.

Walsh S J 2004. Biphasic waveforms for internal and external atrial defibrillation (MD thesis). Queen's University of Belfast, UK.

Wolf P A, Abbott R D and Kannel, W B 1991. Atrial fibrillation as an independent risk factor for stroke: the Framingham Study. Stroke 22: 983-8.

Wu X, Weng Y, Sun S, Weil M H and Tang W 2010. Novel rectangular biphasic and monophasic waveforms delivered by a radiofrequency-powered defibrillatorcompared with conventional capacitorbased waveforms in transvenous cardioversion of atrial fibrillation. Europace 122: A81.

Young C, Bisera J, Gehman S, Snyder D, Tanq W and Weil M H 2004. Amplitude spectrum area: measuring the probability of successful defibrillation as applied to human data. Crit Care Med. 32: S356-8. 